\title{
RETRACTED CHAPTER: Reinstating Floyd-Steinberg: Improved Metrics for Quality Assessment of Error Diffusion Algorithms
}

\author{
Sam Hocevar ${ }^{1}$ and Gary Niger ${ }^{2}$ \\ ${ }^{1}$ Laboratoire d'Imagerie Bureautique et de Conception Artistique \\ 14 rue de Plaisance, Paris, France \\ 2143 Rolloffle Avenue, Tarzana, California 91356 \\ sam@hocevar.net, gary_niger@gnaa.us
}

\begin{abstract}
In this contribution we introduce a little-known prope af error diffusion halftoning algorithms which we call error diffy iion a placement. By accounting for the inherent sub-pixel displacement qused by the error propagation, we correct an important flaw ir $\mathrm{m}$ st metrics used to assess the quality of resulting halftones. We find the metrics to usually highly underestimate the quality of error diffus in comparison to more modern algorithms such as direct binary earch. Using empirical observation, we give a method for creating mp atronally efficient, image-independent, model-based metrics for this ality assessment. Finally, we use the properties of error diff sion displacement to justify Floyd and Steinberg's well-known choice of yorithm coefficients.
\end{abstract}

Keywords: halftoning, error diffuss in age quality, human visual system, color quantization.

\section{Introduction}

Image dithering is the proc ess of reducing continuous-tone images to images with a limited number of availabusours. Applications vary tremendously, from laser and ink-jet printing to $a_{n_{1}}$ ray on small devices such as cellphones, or even the design of banknotes

Countless peth ds nave been published for the last 40 years that try to best address the plem of colour reduction. Comparing two algorithms in terms of speed or memory usage is often straightforward, but how exactly a halftoning algorithn oer.orms quality-wise is a far more complex issue, as it highly depends on the dispray device and the inner workings of the human eye.

T. ugh this document focuses on the particular case of bilevel halftoning, most of our results can be directly adapted to the more generic problem of colour reduction.

\section{Halftoning Algorithms}

The most ancient halftoning method is probably classical screening. This highly parallelisible algorithm consists in tiling a dither matrix over the image and

The original version of this chapter was retracted: The retraction note to this chapter is available at https://doi.org/10.1007/978-3-540-69905-7_71

A. Elmoataz et al. (Eds.): ICISP 2008, LNCS 5099, pp. 38 45, 2008.

(C) Springer-Verlag Berlin Heidelberg 2008, corrected publication 2021 
using its elements as threshold values. Classical screening is known for its structural artifacts such as the cross-hatch patterns caused by Bayer ordered dither matrices [1]. However, modern techniques such as the void-and-cluster method [2, 3] allow to generate screens yielding visually pleasing results.

Error diffusion dithering, introduced in 1976 by Floyd and Steinberg [4, tries to compensate for the thresholding error through the use of feedback. Typically applied in raster scan order, it uses an error diffusion matrix such as the following one, where $x$ denotes the pixel being processed:

$$
\frac{1}{16}\left|\begin{array}{rrr}
-x & 7 \\
3 & 5 & 1
\end{array}\right|
$$

Though efforts have been made to make error diffusion parallelisab -5, it is generally considered more computationally expensive than screen, ng, but carefully chosen coefficients yield good visual results [6].

Model-based halftoning is the third important algorithm co gory. It relies on a model of the human visual system (HVS) and attemp to minimise an error value based on that model. One such algorithm is irectbinary seach (DBS) [10], also referred to as least-squares model-based al to ring (LSMB) 16].

HVS models are usually low-pass filters. Nasanen a], Analoui and Allebach [10] found that using Gaussian models gave v sually pleasing results, an observation confirmed by independent visual perceptron studies [11].

DBS yields halftones of impressive rali However, despite efforts to make it more efficient [12, it suffers from its rge computational requirements and error diffusion remains a more wide as d technique.

\section{Error Diffusion Displacement}

Most error diffusion imply entations parse the image in raster scan order. Boustrophedonic (sernen ine) scanning has been shown to cause fewer visual artifacts [7, but other mo complex processing paths such as Hilbert curves [8] are seldom used a o ey do not improve the image quality significantly.

Intuitifely, as the error is always propagated to the bottom-left or bottomright of (Ch pixel (Fig. 1), one may expect the resulting image to be slightly transle ted. This expectation is confirmed visually when rapidly switching betwee an error diffused image and the corresponding DBS halftone.

This small translation is visually innocuous but we found that it means a lot in terms of error computation. A common way to compute the error between an image $h_{i, j}$ and the corresponding binary halftone $b_{i, j}$ is to compute the mean square error between modified versions of the images, in the form:

$$
E(h, b)=\frac{\left(\left\|v * h_{i, j}-v * b_{i, j}\right\|_{2}\right)^{2}}{w h}
$$

where $w$ and $h$ are the image dimensions, $*$ denotes the convolution and $v$ is a model for the human visual system. 

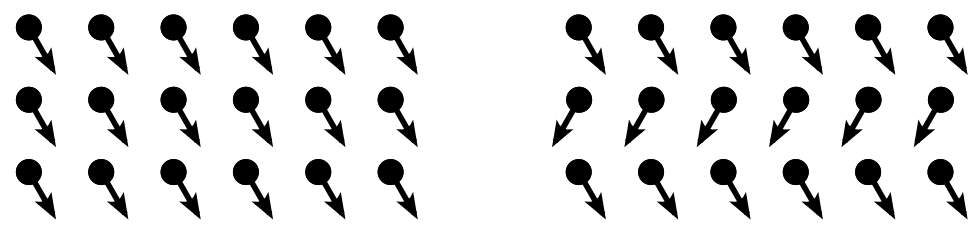

Fig. 1. Floyd-Steinberg error diffusion direction in raster scan (left) and serpentine scan (right)

To compensate for the slight translation observed in the halftone, we following error metric instead:

$$
E_{d x, d y}(h, b)=\frac{\left(\left\|v * h_{i, j}-v * t_{d x, d y} * b_{i, j}\right\|_{2}\right)^{2}}{w h}
$$

where $t_{d x, d y}$ is an operator which translates the image along $0(d x, d y)$ vector. By design, $E_{0,0}=E$.

A simple example can be given using a Gaussian z'VS nodel:

Finding the second filter is then straightforwa

$$
v(x, y)=e^{\frac{x^{2}+y^{2}}{2 \sigma^{2}}}
$$

$$
\left(v * t_{d x, d y}\right)(x, y)=\frac{(-d x)^{2}+(y-d y)^{2}}{2 \sigma^{2}}
$$

Experiments show that for a gixen a age and a given corresponding halftone, $E_{d x, d y}$ has a local minimum a'm t always away from $(d x, d y)=(0,0)$ (Fig. 2). Let $E$ be an error metric where t/s remains true. We call the local minimum $E_{\text {min }}$ :

$$
E_{\min }(h, b)=\min _{d x, d y} E_{d x, d y}(h, b)
$$

For instance, a "oyd-Steinberg dither of Lena with $\sigma=1.2$ yields a per-pixel mean square 6 or of $3.67 \times 10^{-4}$. However, when taking the displacement into account the error becomes $3.06 \times 10^{-4}$ for $(d x, d y)=(0.165,0.293)$. The new, correctea rror is significantly smaller, with the exact same input and output im res

Ex priments show that the corrected error is always noticeably smaller except in the case of images that are already mostly pure black and white. The experiment was performed on a database of 10,000 images from common computer vision sets and from the image board 4chan, providing a representative sampling of the photographs, digital art and business graphics widely exchanged on the Internet nowadays 13 .

In addition to the classical Floyd-Steinberg and Jarvis-Judice-Ninke kernels, we tested two serpentine error diffusion algorithms: Ostromoukhov's simple error diffusion [15, which uses a variable coefficient kernel, and Wong and Allebach's optimum error diffusion kernel [14]: 

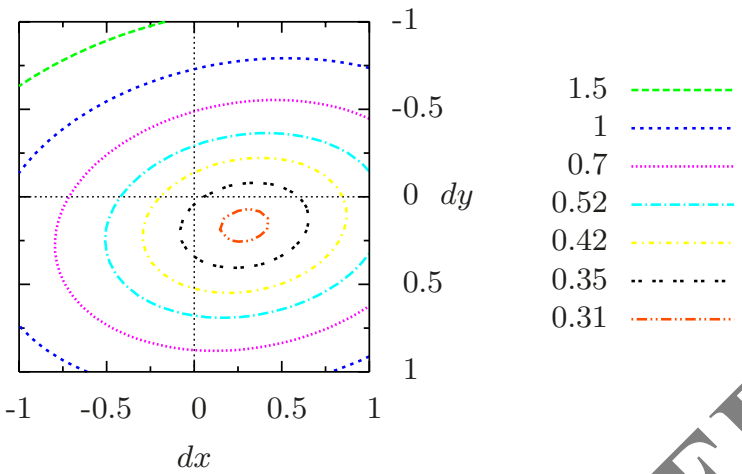

Fig. 2. Mean square error for the Lena image. $v$ is a simple $11 \times 11 \mathrm{Gar}$ (ss) convolution kernel with $\sigma=1.2$ and $(d x, d y)$ vary in $[-1,1] \times[-1,1]$

\begin{tabular}{|l|c|c|}
\hline & $E \times 10^{4}$ & \\
\hline raster Floyd-Steinberg & 3.7902 & $10^{4}$ \\
\hline raster Ja-Ju-Ni & 9.7073 & $\mathbf{8 . 6 3 4 9}$ \\
\hline Ostromoukhov & 4.68 & \\
\hline optimum kernel & $\mathbf{5} 209$ & 6.4783 \\
\hline
\end{tabular}

We clearly see that usual metwes 'nd restimate the quality of error-diffused halftones, especially in raster 0 ๆ. A gorithms such as direct binary search, on the other hand, do not suffer from, his bias since they are designed to minimise the very error induced by he HVS model.

\section{An Image- ndependent Corrected Quality Metric for Error-Dith sed Halftones}

We hav seen that for a given image, $E_{\text {min }}(h, b)$ is a better and fairer visual error mea urement than $E(h, b)$. However, its major drawback is that it is highly con $\mathrm{pu}$-tionally expensive: for each image, the new $(d x, d y)$ values need to be calcu ted to minimise the error value.

Fortunately, we found that for a given raster or serpentine scan error diffusion algorithm, there was often very little variation in the optimal $(d x, d y)$ values (Fig. 3 and 4).

For each algorithm, we choose the $(d x, d y)$ values at the histogram peak and we refer to them as the algorithm's displacement, as opposed to the image's displacement for a given algorithm. We call $E_{\text {fast }}(h, b)$ the error computed at $(d x, d y)$. As $E_{\text {fast }}$ does not depend on the image, it is a lot faster to compute than $E_{m i n}$, and as it is statistically closer to $E_{m i n}$, we can expect it to be a better error estimation than $E$ : 

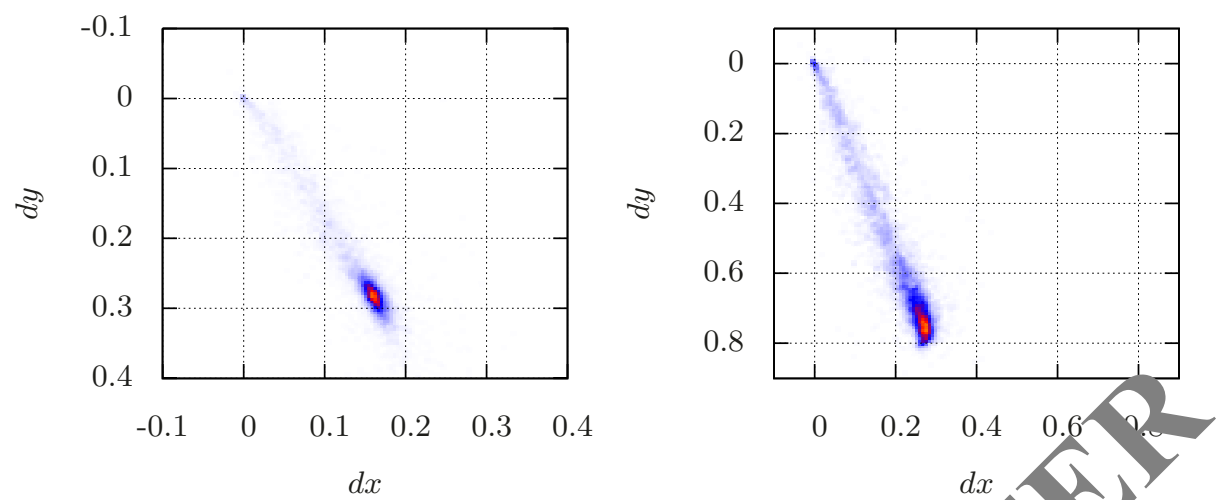

Fig. 3. Error diffusion displacement histograms for the raster Floy A- einberg (left) and raster Jarvis, Judis and Ninke (right) algorithms applied the corp, is of 10,000 images
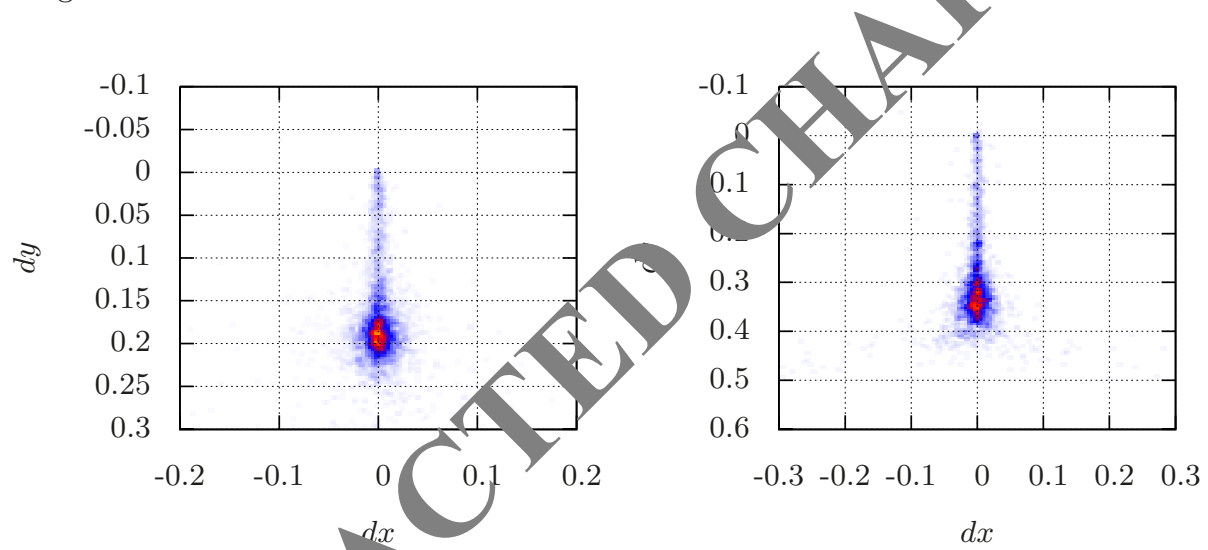

Fig. 4. Error diffusion di.placement histograms for the Ostromoukhov (left) and optimum kernel (right a corit $\mathrm{hms}$ applied to a corpus of 10,000 images

\begin{tabular}{|c|c|c|c|c|c|}
\hline & $E \times 10^{4}$ & $E_{\min } \times 10^{4}$ & $d x$ & $d y$ & $E_{\text {fast }} \times 10^{4}$ \\
\hline raster vi $\overline{\text { dy }}$ Steinberg & 3.7902 & 3.1914 & 0.16 & 0.28 & 3.3447 \\
\hline raste Ja, Ju-Ni & 9.7013 & 6.6349 & 0.26 & 0.76 & 7.5891 \\
\hline Os omoukhov & 4.6892 & 4.4783 & 0.00 & 0.19 & 4.6117 \\
\hline optinnum kernel & 7.5209 & 6.5772 & 0.00 & 0.34 & 6.8233 \\
\hline
\end{tabular}

\section{Using Error Diffusion Displacement for Optimum Kernel Design}

We believe that our higher quality $E_{\text {min }}$ error metric may be useful in kernel design, because it is the very same error that admittedly superior yet computationally expensive algorithms such as DBS try to minimise. 
Our first experiment was a study of the Floyd-Steinberg-like 4-block error diffusion kernels. According to the original authors, the coefficients were found "mostly by trial and error" 4]. With our improved metric, we now have the tools to confirm or infirm Floyd and Steinberg's initial choice.

We chose to do an exhaustive study of every $\frac{1}{16}\{a, b, c, d\}$ integer combination. We deliberately chose positive integers whose sum was 16: error diffusion coefficients smaller than zero or adding up to more than 1 are known to be unstable [17, and diffusing less than $100 \%$ of the error causes important loss of detail in the shadow and highlight areas of the image.

We studied all possible coefficients on a pool of 3,000 images with ap-rror metric $E$ based on a standard Gaussian HVS model. $E_{\min }$ is only given an indication and only $E$ was used to elect the best coefficients:
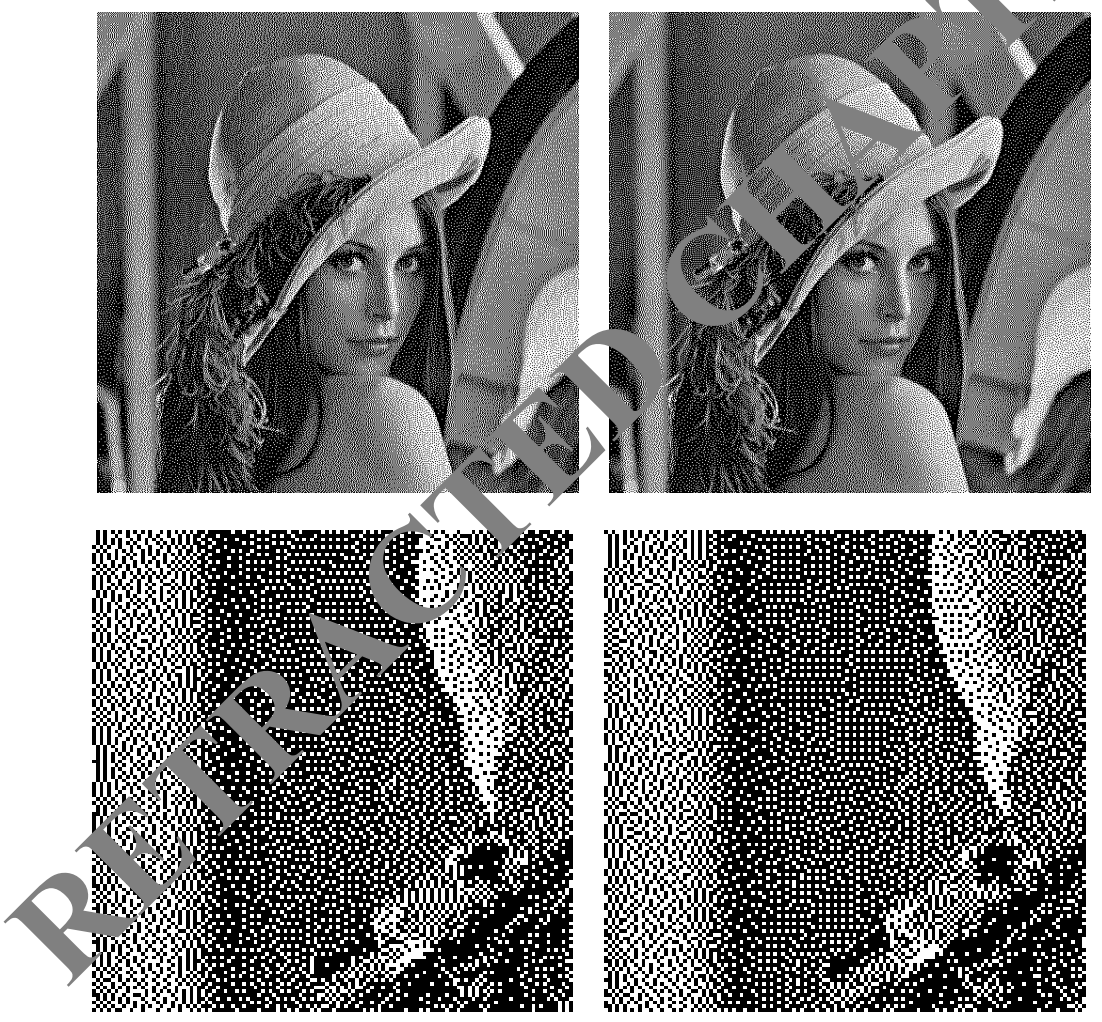

Fig. 5. Halftone of Lena using serpentine error diffusion (left) and the optimum coefficients $\frac{1}{16}\{7,4,5,0\}$ (right) that improve on the standard Floyd-Steinberg coefficients in terms of visual quality for the HVS model used in section 3. The detail (bottom) shows fewer structure artifacts using optimum coefficients. 


\begin{tabular}{|c|c|c|c|}
\hline rank & coefficients & $E \times 10^{4}$ & $E_{\min } \times 10^{4}$ \\
\hline 1 & 7360 & 4.65512 & 3.94217 \\
\hline 2 & 8350 & 4.65834 & 4.03699 \\
\hline \hline 5 & 7351 & 4.68588 & 3.79556 \\
\hline \hline 18 & 6352 & 4.91020 & 3.70465 \\
\hline$\ldots$ & $\ldots$ & $\ldots$ & $\ldots$ \\
\hline
\end{tabular}

The exact same operation using $E_{\text {min }}$ as the decision variable yields very different results. Similarly, $E$ is only given here as an indication:

\begin{tabular}{|c|c|c|c|}
\hline rank & coefficients & $E_{\min } \times 10^{4}$ & $E \times 10^{4}$ \\
\hline 1 & 6352 & 3.70465 & 4.91020 \\
\hline 2 & 7351 & 3.79556 & 4.6858 \\
\hline \hline 15 & 7360 & 3.94217 & 4.65512 \\
\hline \hline 22 & 8350 & 4.03699 & 4.6580 \\
\hline$\ldots$ & $\ldots$ & $\cdots$ & \\
\hline
\end{tabular}

Our improved metric allowed us to confirm that the original Floyd-Steinberg coefficients were indeed amongst the best pos 1 le for raster scan. More importantly, using $E$ as the decision var ab may have elected $\frac{1}{16}\{7,3,6,0\}$ or $\frac{1}{16}\{8,3,5,0\}$, which are in fact poor cho

For serpentine scan, however, ou mprment suggests that $\frac{1}{16}\{7,4,5,0\}$ is a better choice than the Floyd-S inbe, coefficients that have nonetheless been widely in use so far (Fig. 5)

\section{Conclusion}

We have disclose ateresting property of error diffusion algorithms allowing to more prec. ly mysure the quality of such halftoning methods. Having showed that such qualis is often underestimated by usual metrics, we hope to see even more do splnent in simple error diffusion methods.

Co frm 1g Floyd and Steinberg's 30-year old "trial-and-error" result with our wor to wly the beginning: future work may cover more complex HVS models, for ins,ance by taking into account the angular dependance of the human eye [18. We plan to use our new metric to improve all error diffusion methods that may require fine-tuning of their propagation coefficients.

\section{References}

1. Bayer, B.: Color imaging array. U.S. patent 3,971,065 (1976)

2. Ulichney, R.A. (Digital Equipment Corporation), Void and cluster apparatus and method for generating dither templates. U.S. patent 5,535,020 (1992) 
3. Ancin, H., Bhattacharjya, A., Shu, J. (Seiko Epson Corporation), Void-and-cluster dither-matrix generation for better half-tone uniformity. U.S. patent $6,088,512$ (1997)

4. Floyd, R.W., Steinberg, L.: An adaptive algorithm for spatial grey scale. Proceedings of the Society of Information Display 17, 75-77 (1976)

5. Metaxas, P.: Optimal Parallel Error-Diffusion Dithering. In: Color Imaging: DeviceIndep. Color, Color Hardcopy, and Graphic Arts IV, Proc. SPIE, vol. 3648, pp. 485-494 (1999)

6. Kite, T.D.: Design and Quality Assessment of Forward and Inverse Error-Diffusion Halftoning Algorithms. PhD thesis, Dept. of ECE, The University of Texas at Austin, Austin, TX (August 1998)

7. Ulichney, R.: Digital Halftoning. MIT Press, Cambridge (1987)

8. Velho, L., Gomes, J.: Digital halftoning with space-filling curves. In Comp, Ater Graphics (Proceedings of SIGGRAPH 1991), vol. 25(4), pp. 81-90

9. Nasanen, R.: Visibility of halftone dot textures. IEEE Trans. Syst $\mathbb{N}$ ๆ. Cyb. 14(6), 920-924 (1984)

10. Analoui, M., Allebach, J.P.: Model-based halftoning using diro binary search. In: Proc. of SPIE/IS\&T Symp. on Electronic Imaging Sciel anc Tech., San Jose, CA, February 1992, pp. 96-108 (1992)

11. McNamara, A.: Visual Perception in Realistic Imag Sy he Computer Graphics Forum 20(4), 211-224 (2001)

12. Bhatt, et al.: Direct Binary Search with Adar tive Searich and Swap, http://www . ima.umn.edu/2004-2005/MM8.1-10.05/activ ies/Wu-Chai/halftone.pdf

13. moot, http://www.4chan.org/

14. Wong, P.W., Allebach, J.P.: Optimun ror- iffusion kernel design. In: Proc. SPIE, vol. 3018, pp. 236-242 (1997)

15. Ostromoukhov, V.: A Simple and cie terror-Diffusion Algorithm. In: Proceedings of SIGGRAPH 2001, in AM Computer Graphics, Annual Conference Series, pp. 567-572 (2001)

16. Pappas, T.N., Neuhoff, I L.: Least-squares model-based halftoning. In: Proc. SPIE, Human Vision, Visual Pr and Digital Display III, San Jose, CA, February 1992, vol. 1666 , pp. 165-1/0 1992)

17. Eschbach, R., F Z.,Knox, K.T., Marcu, G.: Threshold Modulation and Stability in Error Diffu onal Processing Magazine 20(4), 39-50 (2003)

18. Sullivan, Mì \%, R., Pios, G.: Image halftoning using a visual model in error diffusion J. Apt. Soc. Am. A 10, 1714-1724 (1993)

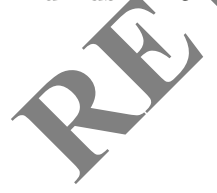

